\title{
Répartition des foraminifères benthiques actuels sur le plateau continental sénégalais
} au sud de Dakar

\author{
Fabrice REDOIS, Jean-Pierre DEBENAY
}

Laboratoire de géologie, EA2644, faculté des sciences, 2, boulevard Lavoisier, 49045 Angers, France Laboratoire d'étude des bio-indicateurs marins (Lebim), Ker Châlon, 85350 Île d'Yeu, France

(Reçu le 19 février 1997, révisé le 2 septembre 1997, accepté le 17 avril 1998)

\begin{abstract}
Recent benthic foraminifers on the continental shelf south of Dakar (Senegal). Two hundred and twentyfour benthic foraminiferal species were recognized in the total assemblages of fifty samples collected from the Senegalese continental shelf south of Dakar (down to a depth of $200 \mathrm{~m}$ ). A factor analysis based on nineteen dominant species shows three main factors. The first two seem to be associated with temperature and its seasonal variations; they are depth-related and determine two main faunal changes. The third factor is substrate-related. The main depth-related species are: i) Cribrononion gerthi, Quinqueloculina akneriana, Quinqueloculina lamarckiana, Cribrolinoides curtus, Elphidium fichtellianum and Textularia sagittula in the coastal zone; ii) Discorbinella bertheloti, Cancris aff. C. sagrum, Textularia truncata, Nonion fabum, Bulimina elegans and Hanzawaia solei in the inner shelf; iii) Trifarina bradyana, Trifarina fornasinii, Uvigerina peregrina and Cassidulina laevigata in the outer shelf. The species that are substrate-related are: i) N. fabum, B. elegans, C. gerthi and H. solei in the fine-grained sands and ii) Lobatula lobatula, C. curtus, T. sagittula and $D$. bertheloti in the coarse-grained sands. The reduction in planktonic percentage and diversity in the outer shelf are related to the upwelling seasonal influence. $\odot$ Elsevier, Paris / Ifremer / Cnrs / Ird
\end{abstract}

\section{recent foraminifer / continental shelf / Atlantic / Senegal}

Résumé - Deux cent vingt-quatre espèces de foraminifères benthiques ont été recensées dans les assemblages totaux de 50 échantillons prélevés sur le plateau continental sénégalais au sud de Dakar (jusqu'à $200 \mathrm{~m}$ de profondeur). Une analyse factorielle portant sur les 19 espèces dominantes met en évidence trois principaux facteurs de répartition pour les peuplements. Les deux premiers sont en relation avec la température des eaux et avec l'amplitude des variations thermiques saisonnières, elles-mêmes étroitement liées à la profondeur. Ils déterminent deux coupures fauniques vers $25 \mathrm{~m}$ et vers $85 \mathrm{~m}$. Le troisième facteur est lié à la nature du sédiment. Les principales espèces dont la répartition est en relation avec la profondeur sont : 1) Cribrononion gerthi, Quinqueloculina akneriana, Quinqueloculina lamarckiana, Cribrolinoides curtus, Elphidium fichtellianum et Textularia sagittula en zone côtière ;2) Discorbinella bertheloti, Cancris aff. C. sagrum, Textularia truncata, Nonion fabum, Bulimina elegans et Hanzawaia solei sur le plateau interne ; 3) Trifarina bradyana, Trifarina fornasinii, Uvigerina peregrina et Cassidulina laevigata sur le plateau externe. Celles dont la répartition dépend de la nature du sédiment sont : 1) N. fabum, B. elegans, C. gerthi et $H$. solei dans les sables fins ; 2) Lobatula lobatula, $C$. curtus, $T$. sagittula et $D$. bertheloti dans les sables grossiers. Sur le plateau externe, la diminution de la diversité et du pourcentage des foraminifères planctoniques semble liée à l'influence des upwellings hivernaux. (C) Elsevier, Paris / Ifremer / Cnrs / Ird

foraminifère actuel / plateau continental / Atlantique / Sénégal 


\section{INTRODUCTION}

Très peu d'études ont été publiées sur les foraminifères benthiques au large des côtes occidentales d'Afrique entre $10^{\circ} \mathrm{N}$ ct $20^{\circ} \mathrm{N}$ [5-7]. À l'exception d'unc radialc étudiée sur le plateau continental [25], les travaux sur le Sénégal au sud de Dakar portent essentiellement sur le passage entre le plateau et la plaine abyssale $[2,9]$. Le travail présenté ici porte sur 50 échantillons prélevés au sud de Dakar entre 10 et $200 \mathrm{~m}$ de profondeur (figure 1). Il se propose : 1) d'établir l'inventaire des espèces ; 2) de déterminer les espèces dominantes et de définir leur répartition cartographique et bathymétrique ; 3 ) de déterminer les principaux facteurs de leur répartition par une analyse factorielle.

\section{ENVIRONNEMENT}

\subsection{Hydrologie}

Le plateau continental étudić est situé au sud de Dakar, entre $14^{\circ} 20^{\prime} \mathrm{N}$ et $14^{\circ} 45^{\prime} \mathrm{N}$ (figure 1). Les masses d'eau qui baignent cette région sont constituées par des eaux tropicales, des eaux guinéennes ou des eaux profondes qui remontent en période d'upwelling. Ces dernières correspondent à un mélange d'eau centrale Nord-Atlantique $(E C N A<20 \%)$ et d'eau centrale Sud-Atlantique (ECSA $>80 \%$ ). En été (juin à octobre), le contre-courant équatorial chaud domine et entraine les eaux guinéennes chaudes et légèrement dessalées jusqu'à la zone étudiée. En hiver (novembre à mai), le courant froid des Canaries

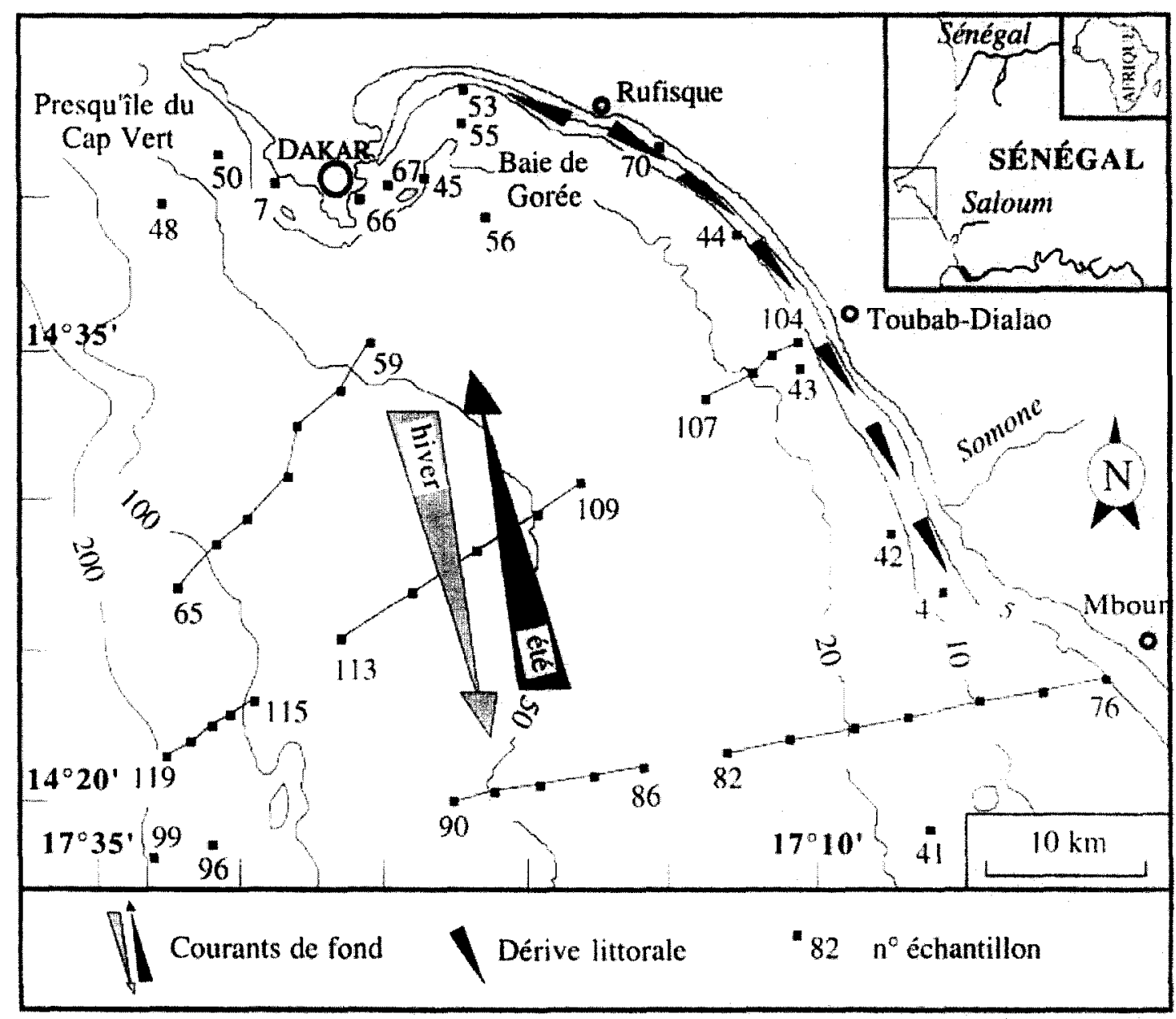

Figure 1. Carte de localisation des prélèvements et des principaux courants $[15,24,27]$

Figure 1. Location map, main currents and sample locations [15, 24, 27]. 
se rapproche des côtes. Son effet est accentué par celui des upwellings qui est maximal en mars-avril [24]. Les houles, dont les effets sont négligeables au-delà de $60 \mathrm{~m}$ $[15,24]$, induisent une dérive littorale sud-est/nord-ouest à l'ouest de Rufisque et nord-ouest/sud-est à l'est de cette ville [26] (figure 1). De 0 à $100 \mathrm{~m}$, les masses d'eaux sont affectées par un courant dirigé vers le Sud en hiver et vers le Nord en été [24]. Sa vitesse au contact du fond est maximale entre 40 et $60 \mathrm{~m}$ de profondeur (environ $\left.10 \mathrm{~cm} \cdot \mathrm{s}^{-1}\right)$.

La salinité est proche de 35,5 sur la plus grande partie du plateau. Seule la zone $0-20 \mathrm{~m}$ est atteinte en été par les eaux guinéennes légèrement dessalées (jusqu'à 34,7). Les eaux sont plus oxygénées en surface $\left(4 \mathrm{~mL} \cdot \mathrm{L}^{-1}\right)$ qu'en profondeur; la teneur de $2 \mathrm{~mL} \cdot \mathrm{L}^{-1}$ est atteinte à $30 \mathrm{~m}$ en hiver et vers $70 \mathrm{~m}$ en été [22]. Les écarts thermiques saisonniers sont maximaux entre 0 et $20 \mathrm{~m}\left(>10^{\circ} \mathrm{C}\right)$; ils sont minimaux au-delà de $100 \mathrm{~m}$ où la température est toujours inférieure à $15^{\circ} \mathrm{C}$ (figure 2). Ces variations saisonnières sont essentiellement dues aux upwellings en hiver $\left(17^{\circ} \mathrm{C}\right.$ en surface) et à la présence d'une thermocline en été $\left(>24^{\circ} \mathrm{C}\right.$ de 0 à $25 \mathrm{~m} ;<19^{\circ} \mathrm{C}$ au-delà de $50 \mathrm{~m}[22,24]$.

\subsection{Géomorphologie - Sédimentologie}

Le plateau continental s'élargit du Nord $(10 \mathrm{~km})$ au Sud $(65 \mathrm{~km})$ (figure 1). Il est accidenté par trois falaises sousmarines plus ou moins continues vers $35 / 45 \mathrm{~m}, 70$ et $90 \mathrm{~m}$ de profondeur. Des bancs rocheux affleurent devant ces falaises ainsi que sur la côte face à Mbour $[8,15,24]$.

En l'absence d'apport fluviatile, les sables qui recouvrent le socle ont des teneurs en pélites $(<50 \mu \mathrm{m})$ rarement supérieures à $10 \%$; elles atteignent toutefois $25 \%$ dans une zone abritée des houles en baie de Gorée. Les éléments terrigènes (quartz) de ces sables sont en grande partie d'origine éolienne $[1,26]$. Les teneurs en carbonates sont souvent supérieures à $60 \%$ compte tenu de l'abondance des bioclastes (mollusques, balanes, foraminifêres et bryozoaires).

Des éléments reliques quaternaires (algues calcaires, bryozoaires, Amphistegina, quartz) sont associés aux dépôts actuels $[11,15]$. Ces éléments usés et parfois épigénisés sont fréquents surtout au-delà de $100 \mathrm{~m}$ [22]. L'héritage quaternaire induit également la présence de trois bandes discontinues, riches en éléments grossiers $(>500 \mu \mathrm{m})$ et plus ou moins parallèles au rivage actuel

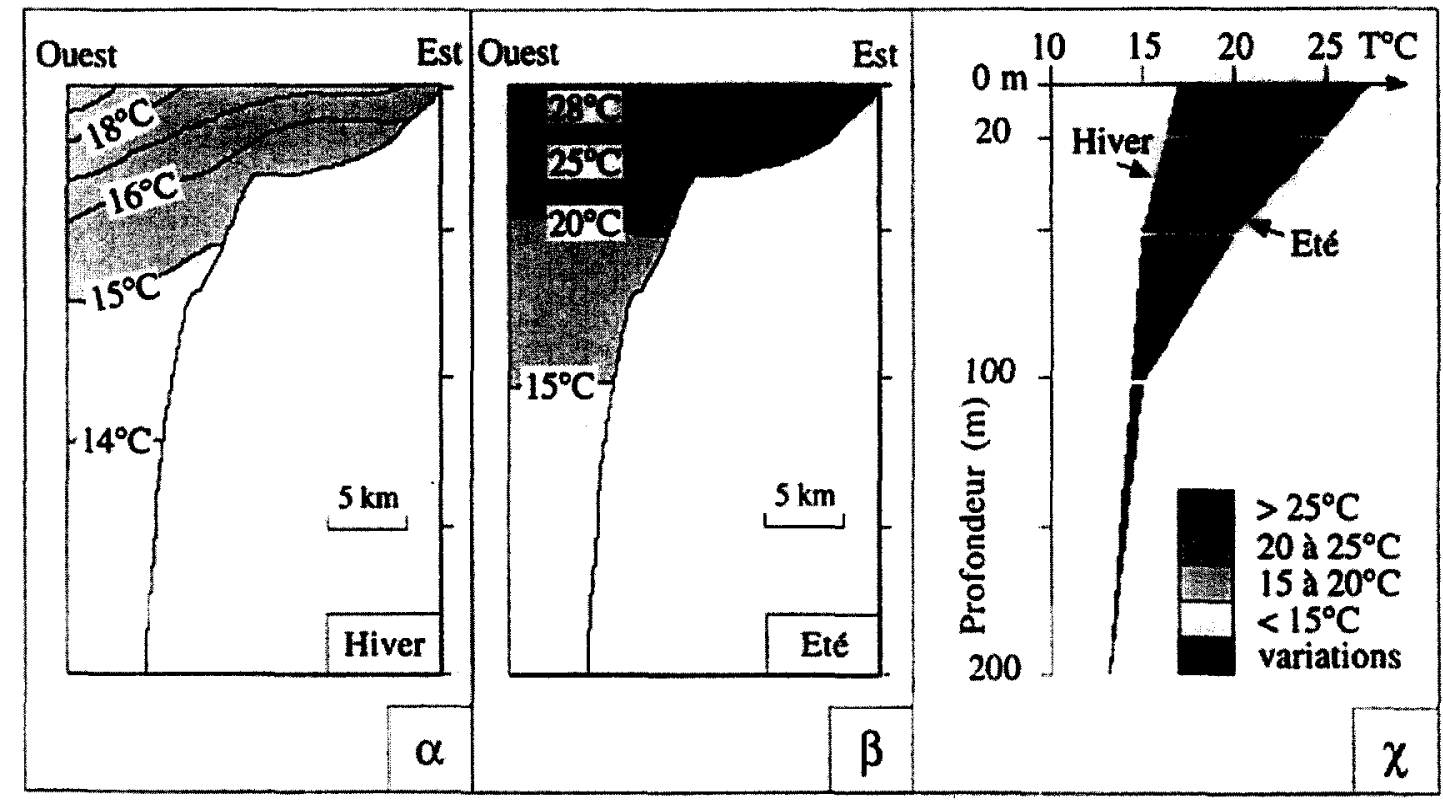

Figure 2. Températures et écarts thermiques saisonniers sur les fonds du plateau continental à proximité de Dakar [22]. ( $\alpha)$ isothermes et profil bathymétrique en hiver ; $(\beta)$ isothermes et profil bathymétrique en été ; $(\chi)$ variations thermiques saisonnières sur le plateau.

Figure 2. Temperatures and seasonal variations in the bottom waters of the continental shelf close to Dakar [22]: ( $\alpha)$ isotherms and depth profile in winter: $(\beta)$ isotherms and depth profile in summer; $(\chi)$ seasonal thermal variations on the shelf. 
(20-30 m, 40-50 m et 80-100 $\mathrm{m}$ de profondeur). Ces bandes et les falaises sous-marines correspondraient à des paléu-rivages édifiés au cours de phases de ralentissement de la transgression postogolienne $[15,26]$.

Six principaux ensembles sédimentaires ont été mis en évidence par Masse [15] : 1) sables fins terrigènes en baie de Gorée (médiane granulométrique ou «MED»: $150 \mu \mathrm{m})$; 2) sables grossiers à débris de balanes autour des zones rocheuses au sud de Toubab-Dialao et sur le pourtour de la presqu'île (MED : $320 \mu \mathrm{m}$ ) [1] ; 3) sables roux grossiers à débris de bryozoaires entre 40 et $50 \mathrm{~m}$ (MED : $480 \mu \mathrm{m}$ ) ; 4) sables fins glauconieux entre 50 et $100 \mathrm{~m}(\mathrm{MED}: 150 \mu \mathrm{m}) ; 5)$ sables du large entre $100 \mathrm{et}$ $200 \mathrm{~m}($ MED : $550 \mu \mathrm{m}) ; 6$ ) sables à Cyclammina à partir de $200 \mathrm{~m}$ (figure 3 ).
À notre connaissance, atcune indication n'est disponible sur la présence éventuelle de végétaux dans la zone étudiée, à l'exception des herbiers signalés en baie de Gorée par Marche-Marchand [15].

\section{MATÉRIEL ET MÉTHODES}

Cinquante échantillons de sédiment superficiel ont été prélevés sur l'ensemble du plateau continental à l'aide d'une drague tubulaire ou d'une drague Charcot (figure 1). Ils ont permis d'effectuer une analyse de la sédimentation biogène [15] avant d'être récemment étudiés pour leur contenu microfaunistique. Seule la fraction comprise entre $125 \mu \mathrm{m}$ et $2 \mathrm{~mm}$ du sédiment brut a été

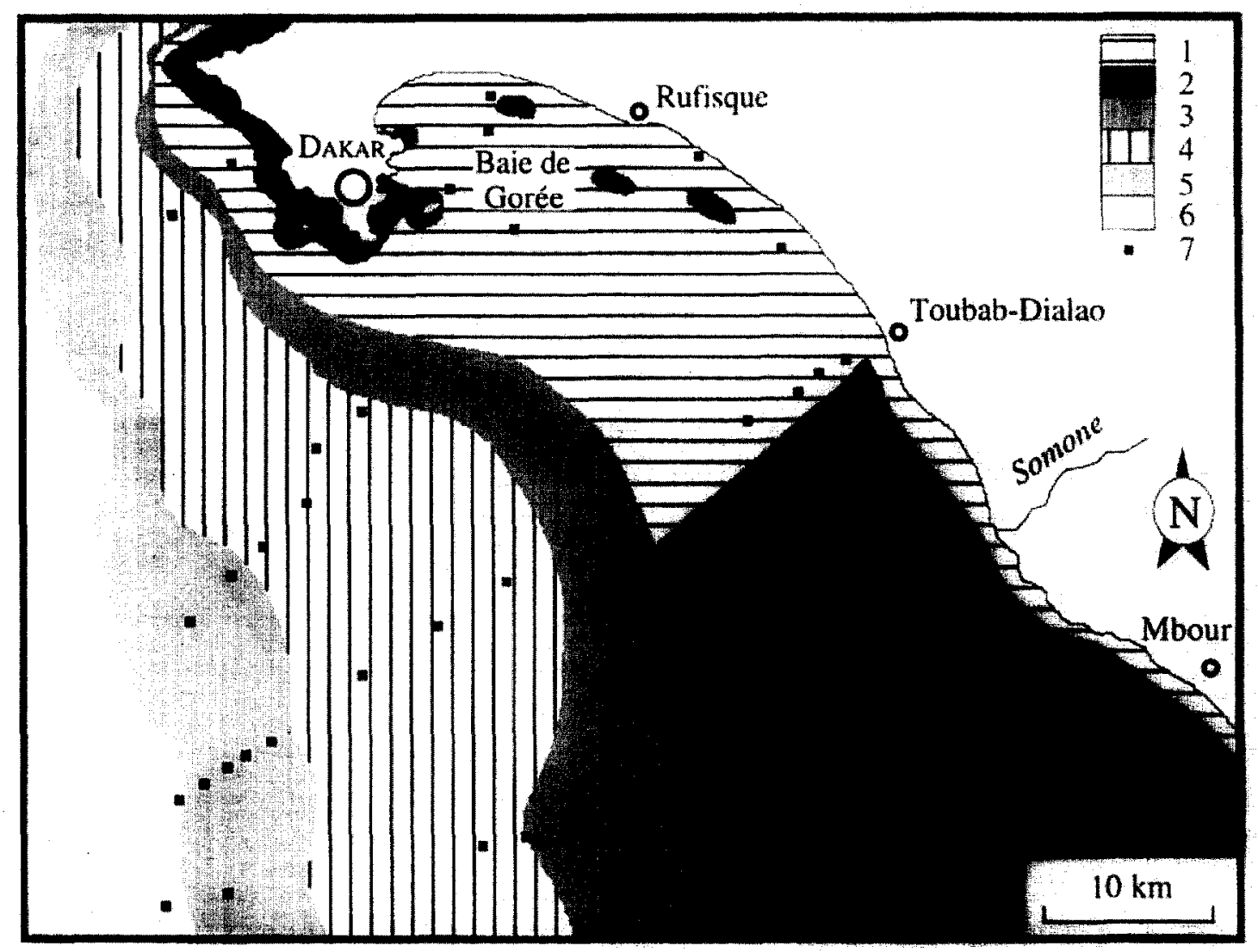

Figure 3. Carte schématique de la répartition des ensembles sédimentaires [15]: (1) sables fins terrigènes; (2) sables à débris de balanes ; (3) sables roux à débris de bryozoaires; (4) sables fins glauconieux ; (5) sables du large ; (6) sables à Cyclammina ; (7) stations de prélèvements. La médiane granulométrique est proche de $150 \mu \mathrm{m}$ pour les ensembles sédimentaires (1) et (4); elle est $\geq$ à $300 \mu \mathrm{m}$ pour les ensembles (2), (3) et (5).

Figure 3. Schematic map of the sedimentary facies distribution [15]: (1) fine-grained terrigeneous sands; (2) Barnacle debris sands; (3) Bryozoan debris russet sands; (4) fine-grained glauconite sands; (5) deeper sands; (6) Cyclammina sands; (7) sample location. The median particle diameter is close to $150 \mu \mathrm{m}$ for sedimentary facies (1) and (4); it is $\geq 300 \mu \mathrm{m}$ for sedimentary facies (2), (3) and (5). 
Tableau I. Liste des espèces classées par ordre alphabétique pour chaque sous-ordre (en gras : espèces dominantes).

Table I. List of benthic foraminiferal species observed (alphabetical order for each suborder); dominant species are in bold text.

\begin{tabular}{|c|c|c|}
\hline Textularilina & Miliollma (suite) & Rotalilina (swite) \\
\hline Alveolophragmium subglobosum (Sars, 1910) & Siphonaperta aff. S. contorta (d'Orbigny, 1846) & Bolivina variabilis (Willianson, 1858) \\
\hline Ammadiscus planorhis HKglund, 1947 & Siphonaperta horrida (Cushman, 1947) & Brizalina goesi (Cushman, 1922) \\
\hline Ammoglobigerina globigeriniformis (Parker \& Jones, 1865) & Spiroloculina antillarum d'Orbigny, 1839 & Brizalina spathulatz (Williamson, 1858) \\
\hline Ammoscalaria pseudospiralis (Williamson, 1858) & Spiroloculina atlantica Cushman, 1947 & Brizalina striatula (Cushman, 1922) \\
\hline Bathysiphon sp. & Spiroloculina circularis Cushman, 1921 & Brizalina subaenariensis (Cushman, 1922) \\
\hline Bigenerina nodosaria d'Orbigny, 1826 & Spiroloculina aff. S. depressa d'Orbigny, 1826 & Bulimina olegans d'Orbigmy, 1826 \\
\hline Cribrostomoides ringens Brady, 1879 & Spiroloculina excavata d'Otbigny, 1826 & Bulimina elongata d'Orbigny, 1826 \\
\hline Cyclammina senegalensis Colom, 1956 & Spiroloculina sp. & Bulimina marginata d'Ortigny, 1826 \\
\hline Deuterammina sp. & Spirophtaimidium acutimargo (Brady, 1884) & Bulimina rostrata Brady, 1884 \\
\hline Eggerelloides scabrus (Willianson, 1858) & Spirophtalmidium sulcata (d'Urbigny, 1826 ) & Bulimina aff. B. striata d'Orblgny, 1843 \\
\hline Gaudnyina cf. G. conica (Parker \& Jones, 1865) & Triloculina tricarinata d'Orbigny, 1826 & Cancris of. C. congolensis Margerel \& Kouyoumontzakis, 1978 \\
\hline Gaudryina redis Wright, 1900 & Triloculina trigonula (Lamarck, 1804) & Cancris aff. C. sagrum (d'Orbigny, 1839) \\
\hline $\begin{array}{l}\text { Jullienella foetida Schlumberger, } 1890 \\
\text { Labrospira jeffreysii (Williamson, 1858) }\end{array}$ & Triloculinella circularis (Bomemann, 1855) & $\begin{array}{l}\text { Cassidella spinescens (Cushman, 1911) } \\
\text { Cassidulina crassa d'Ortigny, } 1839\end{array}$ \\
\hline Lagenammina atlantica (Cushman, 1944) & Spirillinina & Cassidulina laevigata d'Orbigny, 1826 \\
\hline Lepidodeuterammina ochracea (Williamson, 1858) & Patellina cf. P. corrugata Williamson, 1858 & Cassidulinoides braziliensis (Cushman, 1922) \\
\hline Martinoutella communis (d'Orbigny. 1826) & Spirillina vivipara Ehrenberg, 1843 & Chilostomella oolina Schwager, 1878 \\
\hline Nodulina subdentainiformis (Par, 1950) & Spirillina wrightii Heron-Allen \& Earland, 1930 & Chrysalidinella sp. \\
\hline Nouria polymorphinoides Heron-Allen \& Eartand, 1914 & & Cribroelphidium gunteri Cole, 1931 \\
\hline Nouria sp. & Robertinina & Cribroelphidium sp. \\
\hline Paratrochammina rotaliformis Heron-Allen \& Earland, 1911 & Hoeglundina elegans (dorbigny, 1826) & Cribrononion gerthi (Van Vworthuysen, 1957) \\
\hline Placopsilina bradyi Cushman \& Mc Culloch, 1939 & Robertinoides bradyi (Cushman \& Parker, 1936) & Cymbaloporetta bradyi (Cushman, 1915) \\
\hline Psammosphaera fusca Schultze, 1875 & & Discorbinella berthelot (d'Orbigay, 1839) \\
\hline Remaneica plicata (Terquem, 1876) & Lagenina & Dyocibicides biserialis Cushman \& Valentine, 1930 \\
\hline Reophax calcareus (Cushman, 1947) & Amphicoryna scalaris (Batsch, 1791) & Ehrenbergina undulata Parker, 1953 \\
\hline Reophax scorpiurus Montfort, 1808 & Astacolus crepidulus (Fichtel \& Moll. 1798) & Elphidium aculeatum (d'Ortigny, 1846) \\
\hline Reophax subfusiformis Earland. 1933 & Destalina cuvieri (d'Orbigny, 1826) & Elphidium crispum (Linne, 1758) \\
\hline Saccamminz sp. & Favvlina hexagona (Williamson. 1858) & Elphidiurn fichtellianum (d'Orbigny, 1846) \\
\hline Schizammina furcata Heron-Allen \& Earland, 1929 & Favulina melo (d'Orbigny, 1839) & Elphidjum macellum (Fichtel \& Moll, 1798) \\
\hline Septotrochammina gonzalezi (Seiglie, 1964) & Favulina squamosa (Montagu, 1803) & Eponides repandus (Fichtel \& Moll, 1798) \\
\hline Siphotextularia crispata (Brady, 1884) & Fissurina lucida (Williamson, 1848) & Fursenkoina complanata (Egger, 1895) \\
\hline Siphorexwularia rolshauseni Phleger \& Parker, 1951 & Fissurina cf. F. marginata (Montagu, 1803) & Furscnkoina fusiformis (Williamson, 1858) \\
\hline Spiroplectinella wrighti (Silvestri, 1903) & Fissurina sp. & Gavelinopsis praegeri (Heron-Allen \& Earland, 1913) \\
\hline Tetragonostomina cf. T. foliacea (Heron-Allen \& Earland, 1915) & Glandulina laevigata (d'Orbigny, 1826) & Gavelinopsis cf. G. transluscens (Phleger \& Parker, 1951) \\
\hline Tetragonostomina cf. T. mexicana (Cushman, 1922) & Globulina gibba (đ'Orbigny. 1826) & Glabratella patelliformis (Brady, 1884) \\
\hline Tetragenostomina rhombiformis Mikhalevich, 1975 & Globulina myristiformis (Williamson, 1858) & Giabratella sp. \\
\hline Textularia cf T. calva Lalicker, 1935 & Grigelis guttifexa (dOrbigny, 1846) & Globobulimina auriculata (Bailey, 1851) \\
\hline Textularia candeiara d'Orbigny, 1839 & Grigelis of. G. pyrulus (d'Orbigny, 1826) & Globobulimina australiensis Collins. 1958 \\
\hline Textularia lateralis Lalicker. 1935 & Hemirobulina glabra (d'Otbigny, 1826) & Globocassidulina subglobosa (Brady, 1881) \\
\hline Textularia pseudogramen Chapman \& Parr, 1937 & Hemirobulina obesa (Cushman, 1923) & Gyroidina umbonata (Silvestri, 1898) \\
\hline Textularia pseudongosa (Lacroix, 1932) & Hyalinonetrion aff. H. clavatum (Williumson, 1846) & Hanzawaia solei Colom, 1957 \\
\hline Textularia sngittula Detrance, 1824 & Laevidentalina communis (d'Orbigny, 1826) & Heterolepa pseudoungeriana (Cushman, 1922) \\
\hline Textularia truncata Fögluend, 1947 & Laevidentalina filiformis (d'Orbigny, 1826) & Hyalinea balthica (Schnotter, 1783) \\
\hline Trochammina cf. T. grisea Earland, 1914 & Laevidentalina intorta (Dervieux, 1894) & Lobatula flechteri (Galloway \& Wissier, 1927) \\
\hline & Lagena aspera Reuss, 1862 & Lobatula lobatula (Walker \& Jacob, 1798) \\
\hline Mitiolina & Lagena costareticulata Debenay, 1988 & Loxostomina mayori (Cushman, 1922) \\
\hline Biloculinella depressa (d'Orbigny, 1826) & Lagena laevis (Montagu, 1803) & Lugdunum alatum (Seguenza, 1862) \\
\hline Comuspira foliacea (Philippi, 1844) & Lagena semistriata Wiltianson. 1848 & Neoconorbina nitida (Williamson, 1858) \\
\hline Cornuspira involvens (Reuss, 1850) & Lagena striainterupta Debenay, 1988 & Nonion fahum (Fichtel \& Moll, 1798) \\
\hline Cribrolinoides curtas (Cushman, 1917) & Lagena cf. L. sulcata (Walker \& Jacob. 1798) & Nonionella cordiformis (Costa, 1856) \\
\hline Cycloforina sp. & Lagena sulcata spicata Cushrnan \& Mc Culloch, 1950 & Oridorsalis umbonatus (Reuss, 1851) \\
\hline Edentostomina disparilis (Terquem, 1878) & Lagena williamsoni (Alcock, 1865) & Pararotalia armata (d'Orbigny. 1826) \\
\hline Edentostomina dorsata (Reuss, 1866) & Lenticulina calcar (Linne, 1758) & Planodiscorbis rarescens (Brady, 1884) \\
\hline Edentostomina sp. & Lenticulina cuitrata (Montfort, 1808) & Planorbulina mediterranensis d'Orbigny, 1826 \\
\hline Lachlanella autheriana (d'O-bigny, 1839) & Lenticulina gibba (d'Orbigny, 1839) & Planulina ariminensis d'Ortigny, 1826 \\
\hline Lachlanella bicornis (Walker \& Jacob, 1839) & Lenticulina suborbicularis Parr, 1950 & Poroeponides lateralis (Terquem, 1878) \\
\hline Lachlanella ef. L. boveana d'Orbigny, 1846 & Marginulogsis bradyi (Goes, 1894) & Pseudoeponides falsobeccarii Rouvillois, 1974 \\
\hline Lachlanelin sp. ] & Neolenticulina peregrina (Schwager, 1866) & Rectubulivina bifruns (Brady, 1881) \\
\hline Lachlanella sp. 2 & Nodosaria sp. & Rectuvigerina arquatensis (Papp, 1963) \\
\hline Miliolinella sublineata (Brady, 1884) & Palliolatella orbignyana (Seguenza, 1862) & Rectuvigerina phlegeri Le Calvez. 1958 \\
\hline Miliolinella subrotunda (Montagu, 1803) & Paltiolatella radiatomarginata (Parker \& Jones, 1865) & Reussella spinulosa (Reuss, 1850) \\
\hline Nubeculina sp. & Palliolatella sp. & Rosalina bradyi (Cushman, 1915) \\
\hline Nummoloculina contraria (d'Orbigny, 1846) & Polymorphina sp. & Rosalina globularis d'Orbigny, 1826 \\
\hline Nummulopyrgo globulus (Bomemann, 1855) & Pyramidulina catesbyi (d'Orbigny, 1839) & Rosalina rugosa d'Orbigny, 1839 \\
\hline $\begin{array}{l}\text { Peneroplis sp. } \\
\text { Pseudomassilina sp. }\end{array}$ & $\begin{array}{l}\text { Pytine parthenopeia Montcharmont Zei \& Sgarella, } 1978 \\
\text { Saracenaria italica Defrance, } 1824\end{array}$ & $\begin{array}{l}\text { Sigmavirgulina tortuosa (Brady, 1881) } \\
\text { Siphonina reticulata (Crjzek, 1848) }\end{array}$ \\
\hline Ptychomiliola separans (Brady, 1881) & Säracenaria sp. 1 & Sphaerogypsina globula (Reuss, 1848) \\
\hline Pyrgo ringens (Lamarck, 1804) & Saracenaria sp. 2 & Stomatorbina concentrica (Parker \& Jones, 1864) \\
\hline Quinguetoentina akmeriena d'Orbigny, 1839 & Webbinella hemisphaerico (Parker, Jones \& Brady, 1866) & Trifarina bradyana (Cushman, 1932) \\
\hline Quinqueloculina bicostata d'Orbigny, 1839 & & Trifarina bradyi Cushman, 1923 \\
\hline Quinqueloculina disparilis d'Orbigny, 1893 & Rotaliina & Trifarina elongatastriata (Colom, 1952) \\
\hline Quinqueloculina laevigata d'Orbigny, 1839 & Acervulina inhaerens Schultze, 1854 & Trifarina fornasinti (Selli, 1948) \\
\hline Quinqueloculina lamarckiana d'Orbigny, 1839 & Ammonia beccarii (Linne, 1758) & Uvigerina dirupta Todd, 1948 \\
\hline Quinqueloculina procerapertura Debenay, 1988 & Ammonia parkinsoniana (d'Ortigny, 1839) & Uvigerina meditertanea Hofker, 1932 \\
\hline Quinqueloculina venusta Karrer, 1868 & Ammonia tepida (Cushman, 1926) & Uvigerina peregrina Cushman, 1923 \\
\hline Quinqueloculina vulgaris d'Orbigny, 1826 & Amphistegina gibbosa d'Orbigny, 1839 & Uvigerina sp. \\
\hline Quinqueloculina sp. & Angulogerina angulosa (Williamson, 1858) & Valvulineria minuta Parker, 1954 \\
\hline Sigmoilina elliptica Galloway \& Wissler, 1927 & Asterigerinata mamilla (Williamson, 1858) & \\
\hline Sigmoilopsis proceracollis Mikhalevich, 1988 & Astrononion stelligerum (d'Orbigny, 1839) & \\
\hline
\end{tabular}


utilisée. Les tests de foraminifères ont été séparés par flottage sur tétrachlorure de carbone $\left(\mathrm{CCl}_{4}\right)$. L'étude a porté sur la microfaune totale actuelle, sans prendre en compte les individus fortement usés ou épigénisés, susceptibles d'avoir été transportés ou remaniés. Comme le préconise Murray [18], un examen du résidu a été effectué pour tenir compte des formes actuelles trop lourdes qui n'auraient pas été flottées.

Pour chaque prélèvement : 1) les espèces ont été distinguées selon les règles taxonomiques de Loeblich et Tappan [14] ; 2) plus de 300 individus benthiques ont été comptés comme cela est préconisé par Phleger [21] ; 3) le comptage simultané des formes planctoniques a été effectué.

Ces données ont permis de calculer : 1) les poürcentages relatifs des espèces benthiques par rapport à l'assemblage total (morts et vivants). Le comptage de 300 individus donnerait, selon les récents travaux de Patterson et Fishbein [20], une erreur inférieure à $3 \%$ pour toutes les espèces représentant plus de $5 \%$ du peuplement; 2) l'indice de diversité de Shannon-Wiener $H(S)=$ $-\Sigma p_{i} \log _{e} p_{i}$,; où $p_{i}$ est la proportion de la $1^{\text {re }}$ espèce $[18]$; 3) le pourcentage de formes planctoniques, défini par Lévy et al. [13] comme un indice d'océanité.

Dans cette étude, les espèces benthiques représentant plus de $5 \%$ du peuplement dans au moins quatre échantillons, sont considérées comme dominantes et retenues pour une analyse en composantes principales (ACP). Culver et Buzas [4] ont montré que les analyses statistiques portant sur les espèces dominantes, donnent des résultats aussi significatifs que ceux basés sur l'ensemble des espèces. De plus, la sélection d'espèces dominantes permet d'éliminer les risques d'erreur liés aux rares tests Quinqueloculina procerapertura et Spirophtalmidium transportés ou remaniés et non identifiés comme tels durant les comptages.

Dix-neuf espèces dominantes ont ainsi été retenues pour cette ACP réalisée sur le logiciel STATlab 2.0 pour Macintosh. Seuls les trois premiers facteurs sont considérés ; ils expliquent au total $59 \%$ de la variance. Les éléments pris en compte sont : 1) les coordonnées des échantillons et des espèces sur chaque axe factoriel ; 2) les contributions des échantillons à la variance de chaque facteur; 3 ) les corrélations des espèces dominantes avec chaque facteur (valeurs $>0,1$ ). Les résultats de l'analyse sont comparés aux données environnementales afin d'expliquer la signification des principaux facteurs ainsi déterminés.

Les photographies au microscope électronique à balayage ont été réalisées au service commun de microscopie électronique (SCME) de l'université d'Angers ; elles sont présentées à la même échelle $(\times 75)$ sur toutes les planches.

\section{RÉSULTATS}

\subsection{Les espèces}

Deux cent vingt-quatre espèces ont été distinguées (planche I, 5-7, planche III, 6) dont 23 laissées en nomenclature ouverte (tableau I). D'après la synthèse de Debenay et Basov [5], neuf d'entre elles sont citées pour la première fois sur le plateau continental, entre $10^{\circ} \mathrm{N}$ et $20^{\circ} \mathrm{N}$ : Ammonia parkinsoniana, Elphidium aculeatum (planche I, 3), Palliolatella radiatomarginata (planche III, 5), Lachlanella bicornis, Lenticulina gibba, Hemirobulina obesa (planche III, 4), Marginulopsis bradyi, acutimargo.

Planche I. Espèces de la zone côtière (A).

1 Textularia sagittula (A.sg). 2 Cribrononion gerthi (A.sf). 3 Elphidium aculeatum. 4 Elphidium fichtellianum (A). 5 Cribroelphidium sp. 6 Peneroplis sp. 7 Edentostomina sp. 8 Cribrolinoides curtus (A.sg). 9 Quinqueloculina lamarckiana (A). 10 Quinqueloculina ackneriana (A).

11 Cribroelphidium gunteri, vue latérale.

Plate I. Species of the coastal nearshore zone (A)

1 Textularia sagittula (A.sg). 2 Cribrononion gerthi (A.sf). 3 Elphidium aculeatum. 4 Elphidium fichtellianum (A). 5 Cribroelphidium sp. 6 Peneroplis sp. 7 Edentostomina sp. 8 Cribrolinoides curtus (A.sg). 9 Quinqueloculina lamarckiana (A). 10 Quinqueloculina ackneriana (A). 11 Cribroelphidium gunteri, lateral view.

Les associations déterminées à partir de l'analyse factorielle sont représentées par les lettres suivantes : (A) zone côtière ; (B) plateau ; (B1) plateau interne ; (B2) plateau externe ; (sf) sables fins; (sg) sables grossiers. Les barres d'échelle correspondent à $100 \mu \mathrm{m}$.

The associations recognised from the factor analysis are represented by the following letters: (A) coastal nearshore; (B) shelf; (B1) inner shelf; (B2) outer shelf; (sf): fine-grained sands; (sg) coarse-grained sands. The scale bar corresponds to $100 \mu \mathrm{m}$. 


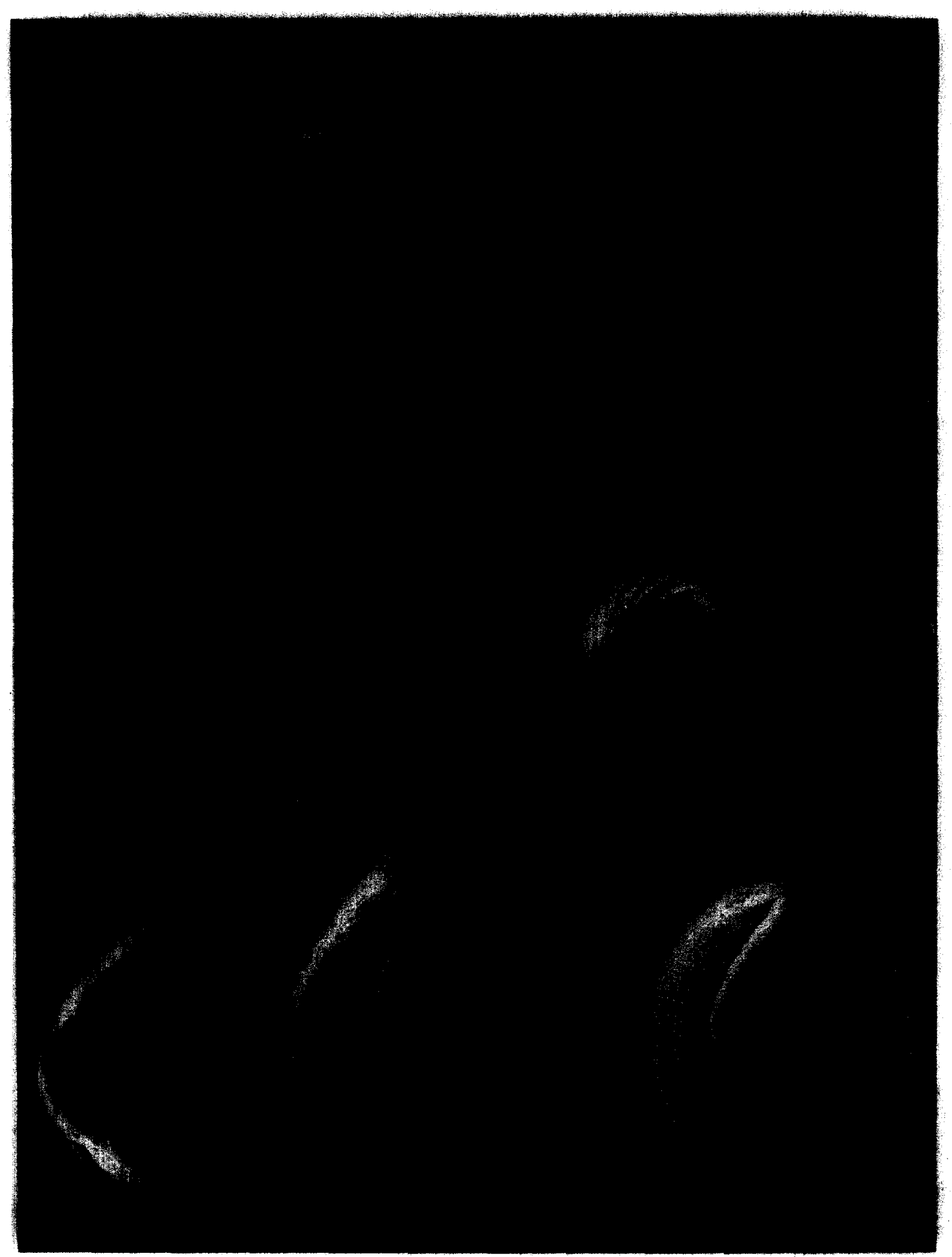




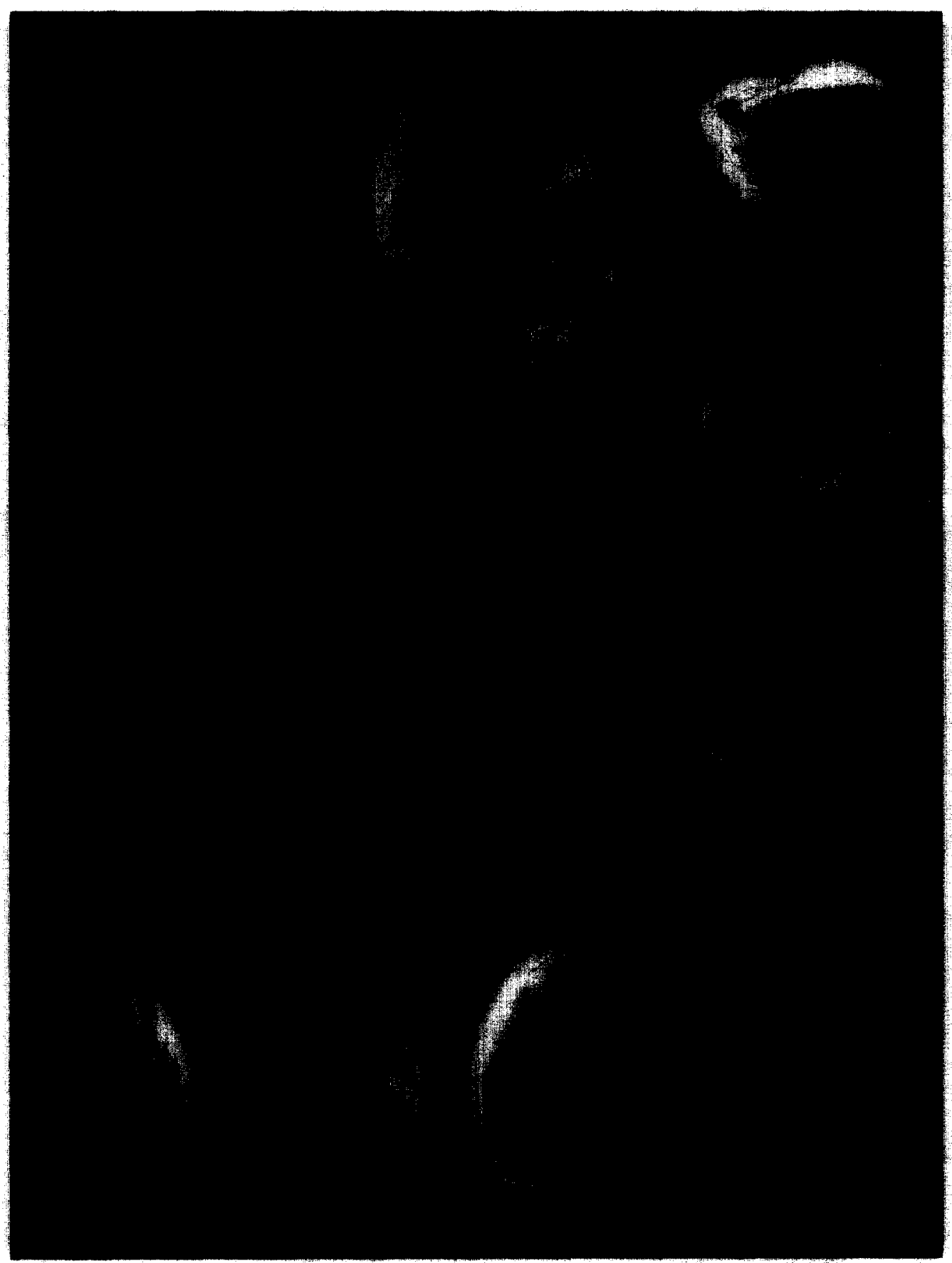

Planche II. Espèces du plateau interne (B1)

1 Cancris aff. C. sagrum (B1). la Spécimen avec dernière loge très développée. 1 b Spécimen avec dernière loge trapue. 2 Textularia truncata (B1). 3 Discorbinella bertheloti (B1.sg). 4 Hanzawaia solei (B1.sf). 5 Bulimina elegans (B1.sf). 6 Bulimina elongata. 7 Nonion fabum (B1.sf). $7 \mathrm{a}-7 \mathrm{~b}$ Spécimens avec dernière loge renflée. $7 \mathrm{c}-7 \mathrm{~d}$ Spécimens avec dernière loge aplatie.

Plate II. Species of the inner shelf (B1)

1 Cancris aff. C. sagrum (B1). 1a Specimen with elongated ultimate chamber. 1b Specimen with thickset ultimate chamber. 2 Textuluria truncata (B1). 3 Discorbinella bertheloti (B1.sg). 4 Hanzawaia solei (B1.sf). 5 Bulimina elegans (B1.sf). 6 Bulimina elongata. 7 Nonion fabum (B1.sf). 7a-7b Specimens with inflated ultimate chamber. 7c-7d Specimens with compressed ultimate chamber. 


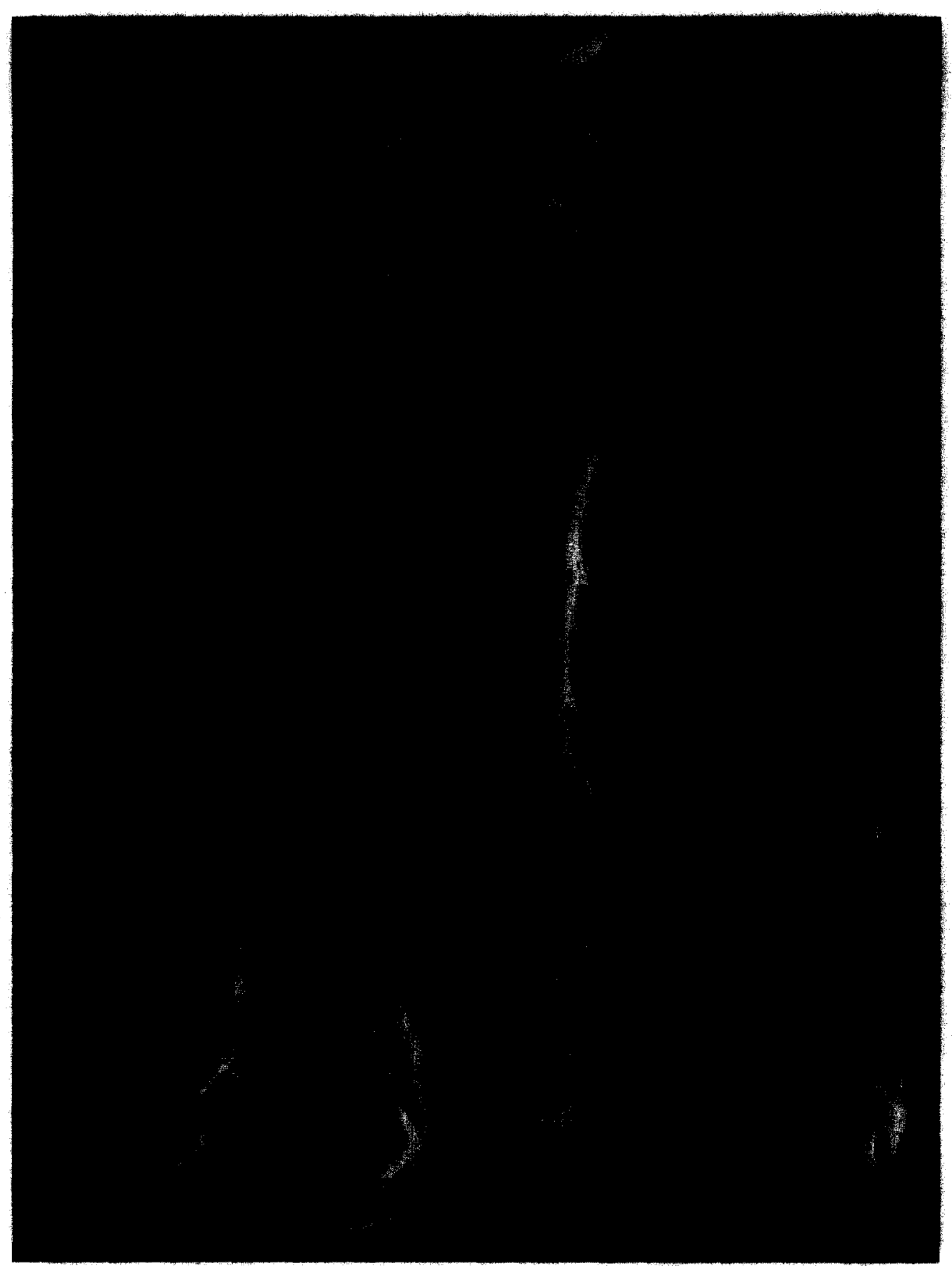

Planche III. Espèces du plateau (B) et du plateau externe (B2)

1 Gaudryina rudis (B). 2 Spiroplectinella wrighti (B). 2a-2b Spécimens avec pointes latérales. 2c Spécimen sans pointe latérale. 3 Lobatula lobatula (B.sg). 4 Hemirobulina obesa. 5 Palliolatella radiatomarginata. 6 Saracenaria sp.1. 7 Trifarina fornasinii (B2). 8 Trifarina bradyana (B2). 9 Uvigerina peregrina (B2). 10 Cassidulina laevigata (B2). 10a Spécimen non caréné. 10b Spécimen caréné.

Plate III. Species of the shelf (B) and of the outer shelf (B2)

1 Gaudryina rudis (B). 2 Spiroplectinella wrighti (B). 2a-2b Specimens with lateral spines. $2 \mathrm{c}$ Specimen without lateral spine. 3 Lobatula lobatula (B.sg). 4 Hemirobulina obesa. 5 Palliolatella radiatomarginata. 6 Saracenaria sp. 1.7 Trifarina fornasinii (B2). 8 Trifarina bradyana (B2). 9 Uvigerina peregrina (B2). 10 Cassidulina laevigata (B2). 10a Non-carinated specimen. 10b Carinated specimen. 

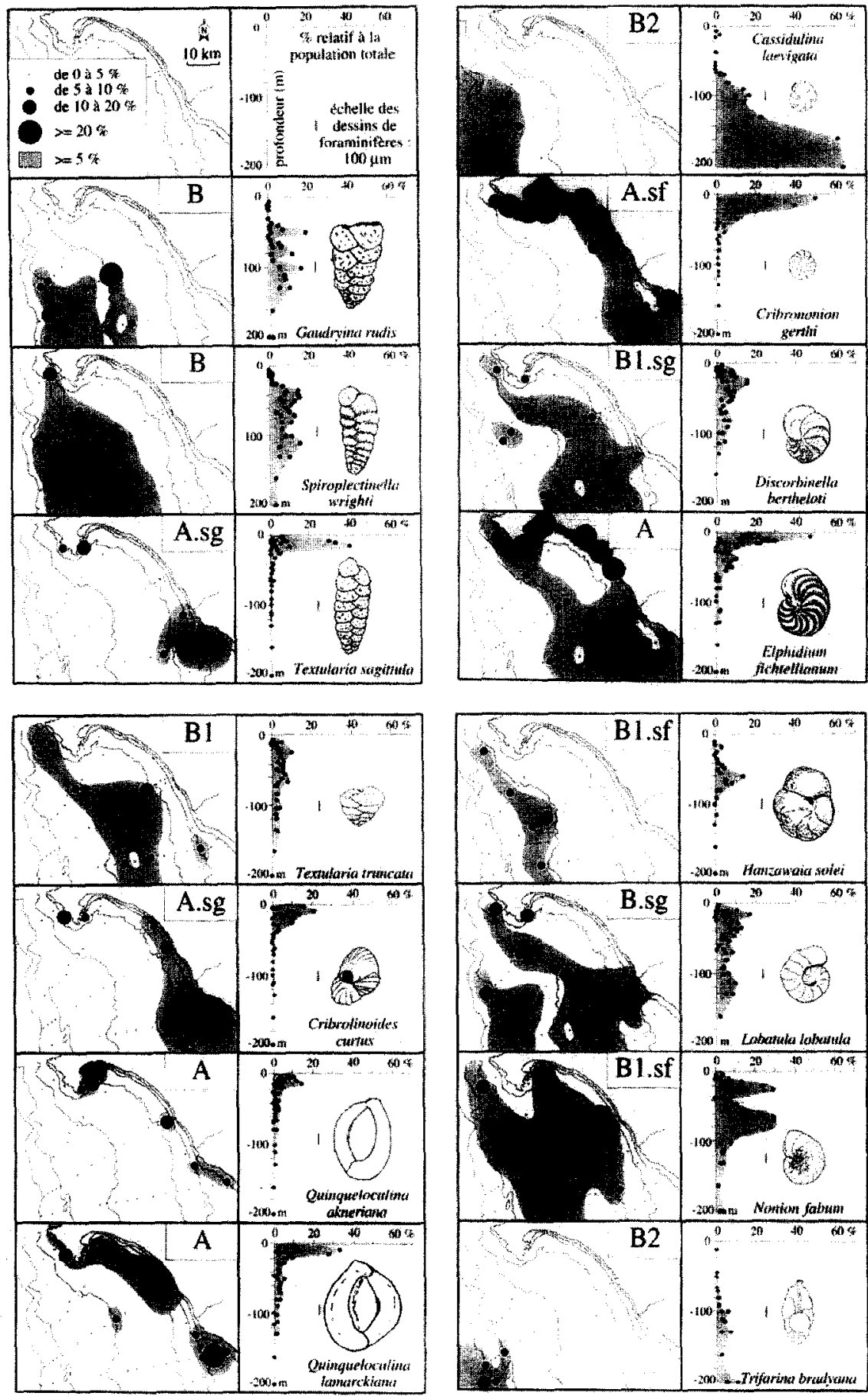

Figare 4. Répartitions cartographique et bathymétrique des 19 espèces dominantes. Les associations déterminées à partir des trois premiers facteurs de l'analyse factorielle sont représentées par les lettres encadrées : (A) zone côtière ; (B) plateau;(B1) plateau interne ; (B2) plateau externe; (sf) sables fins; ( $\mathbf{s g}$ ) sables grossiers.

Figure 4. Geographic and depth distributions of the 19 dominant species. The associations recognized from the three main factors of the factor analysis are represented by letters: (A) coastal zone; (B) shelf; (B1) inner shelf; (B2) outer shelf; (sf) fine-grained sands; (sg) coarse-grained sands. 

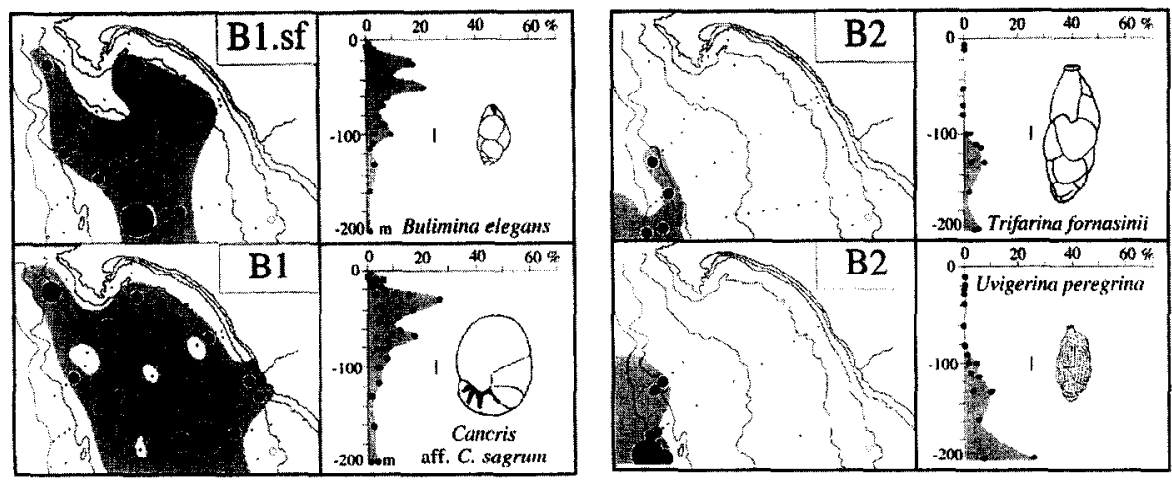

Figure 4. suite.

\subsection{Répartition des espèces dominantes}

Les dix-neuf espèces retenues pour l'analyse factorielle ont une répartition largement influencée par la profondeur (tableau II ; figure 4). Dans certains cas, elle dépend également d'autres facteurs dont l'influence est mise en évidence par: 1) l'irrégularité des répartitions dans une même zone bathymétrique (Textularia sagittula (planche I, 1), Cribrolinoides curtus (planche I, 8) et Elphidium fichtellianum (planche 1,4 ) ; 2) ou l'existence de plusieurs pics de fréquence à des profondeurs variables (Gaudryina rudis (planche III, 1), Spiroplectinella wrighti (planche III, 2), Bulimina elegans (planche II, 5), Cancris aff. C. sagrum (planche II, 4), Lobatula lobatula (planche III, 3) et surtout Nonion fabum (planche II, 7)).

\subsection{Indice de diversité $[H(S)]$}

L'indice de Shannon-Wiener varie de 1,8 à 3,7. Les assemblages les moins diversifiés $[H(S)<2,5]$ sont observés en zone côtière (baie de Gorée et face à Mbour) ainsi qu'entre 160 et $200 \mathrm{~m}$ de profondeur (figure 5). Les assemblages les plus diversifiés $[\mathrm{H}(\mathrm{S})>3,5]$ se trouvent

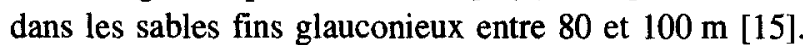
Dans cette zone, la température varie de 15 à $17^{\circ} \mathrm{C}$, la salinité est proche de 35,5 et les teneurs en oxygène sont inférieures à $2 \mathrm{~mL} \cdot \mathrm{L}^{-1}[22]$.

\subsection{Pourcentages de planctoniques}

Le pourcentage des planctoniques varie avec la profondeur (figure 6). Il augmente de la zone côtière $(<10 \%)$ jusqu'à $100 \mathrm{~m}$ (près de $50 \%$ ) puis diminue jusqu'à la limite du domaine étudié (de 20 à $30 \%$ à $200 \mathrm{~m}$ ).

\subsection{Principaux facteurs déterminés par l'analyse factorielle}

Seuls les trois premiers facteurs de répartition des espèces dominantes ont été retenus. Ils expliquent respectivement 25,21 et $13 \%$ de la variance; soit une valeur cumulée proche de $60 \%$ (figures 7,8 et 9 ).

Le premier facteur détermine une coupure faunique vers $25 \mathrm{~m}$ de profondeur (figure 7 ) ; il oppose une association de zone côtière de 0 à $25 \mathrm{~m}$ (planche I) (Cribrononion gerthi (planche I, 2), Quinqueloculina ackneriana (planche I, 9), Quinqueloculina lamarckiana (planche I, 10), C. curtus, E. fichtellianum et $T$. sagittula) à celle du plateau entre 25 et $200 \mathrm{~m}$ (planche III) (S. wrighti, Textularia truncata (planche II, 2), G. rudis, B. elegans, Cancris aff. C. sagrum, Hanzawaia solei (planche II, 4), L. lobatula, Trifarina bradyana (planche III, 8) et Trifarina fornasinii (planche III, 7)).

Le deuxième facteur détermine une deuxième coupure faunique vers $85 \mathrm{~m}$ (figure 8 ); il oppose une association de plateau interne de 25 à $85 \mathrm{~m}$ (planche II) (Discorbinella bertheloti (planche II, 3), Cancris aff. C. sagrum, T. truncata, $N$. fabum, B. elegans et $H$. solei) à celle du plateau externe de 85 à $200 \mathrm{~m}$ (planche III) (T. bradyana, T. fornasinii, Uvigerina peregrina (planche III, 9) et Cassidulina laevigata (planche III, 10)).

Le troisième facteur est lié à la nature du sédiment superficiel qui varie surtout entre la zone côtière et le plateau interne (de 0 à $85 \mathrm{~m}$ ). Deux types d'associations s'y distinguent en fonction des différents ensembles sédimentaires : 1) une association de sables fins (terrigènes ou glauconieux) avec $N$. fabum, B. elegans, C. gerthi et $H$. solei ; 2) une association de sables grossiers (sables bioclastiques à débris de balanes ou sables roux à débris de bryozoaires) avec L. lobatula, C.curtus, $T$. sagittula et D. bertheloti. 
Tableau II. Résultats de l'étude des 50 échantillons : • pourcentages relatifs des 19 espèces dominantes par rapport à l'assemblage total (en grisé : valeurs $\geq 5 \%$; en bas du tableau : limites bathymétriques avec des valeurs $\geq$ à $5 \%$ ou $(20 \%)$; $\bullet$ indices de diversité de Shannon-Wiener $[\mathrm{H}(\mathrm{S})]$; $\bullet$ pourcentages de planctoniques ; $\bullet$ associations d'espèces dominantes déterminées par l'analyse factorielle $(*)$ : (A) zone côtière ; (B) plateau ; (B1) plateau interne ; (B2) plateau externe ; (sf) sables fins : (sg) sables grossiers.

Table II. Study of the 50 samples: $\bullet$ relative percentages of the 19 dominant species (highlighted values are $\geq 5 \%$; bottom of the table: depth limits where values are $\geq 5 \%$ or $\geq 20 \%$ ); $\bullet$ Shannon-Wiener diversity index: H(S); • planktonic percentages; $\bullet$ dominant species associations identified by the factor analysis (*): (A) coastal zone; (B) shelf; (B1) inner shelf; (B2) outer shelf; (sf) fine-grained sands; (sg) coarse-grained sands.

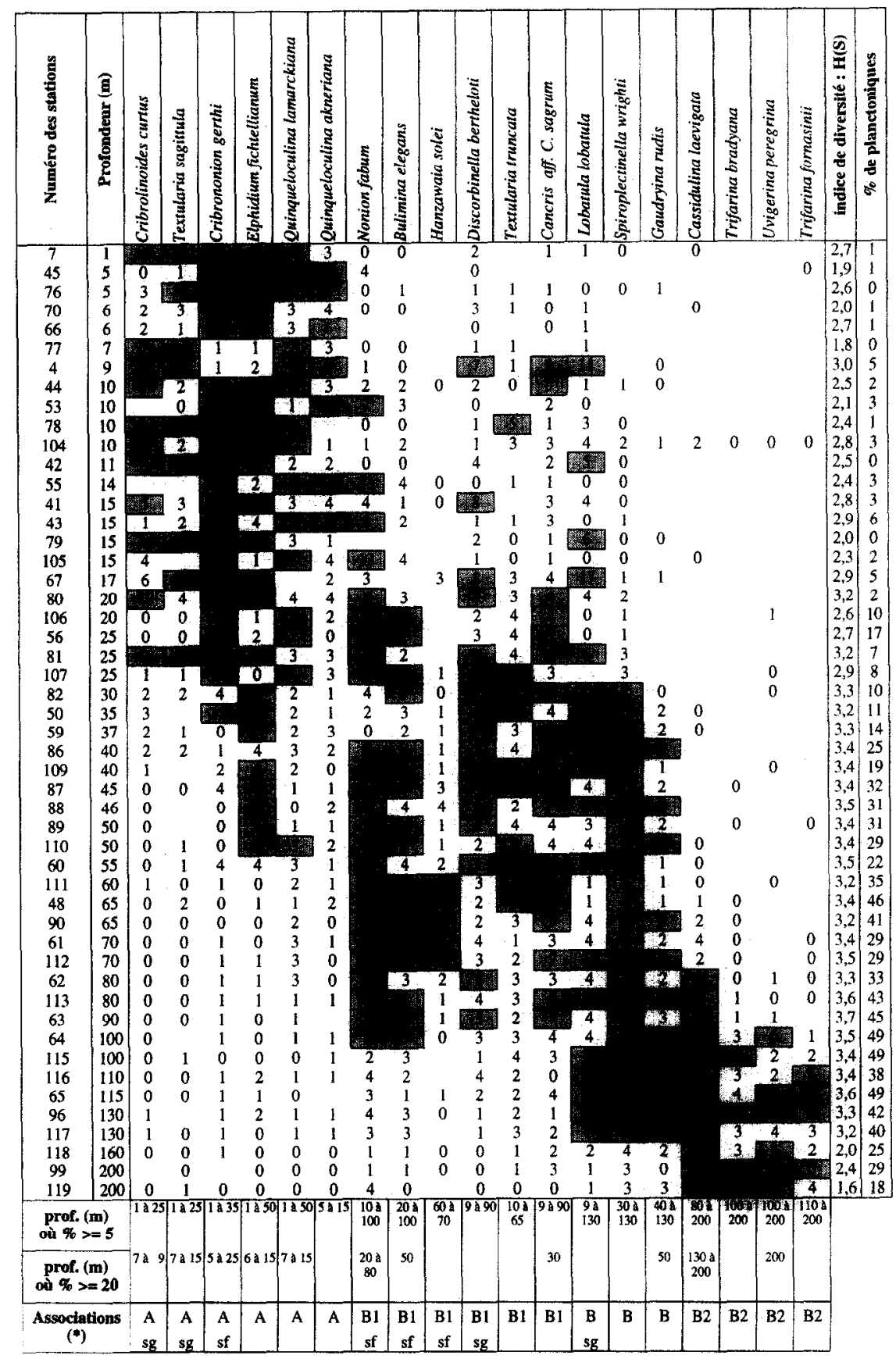




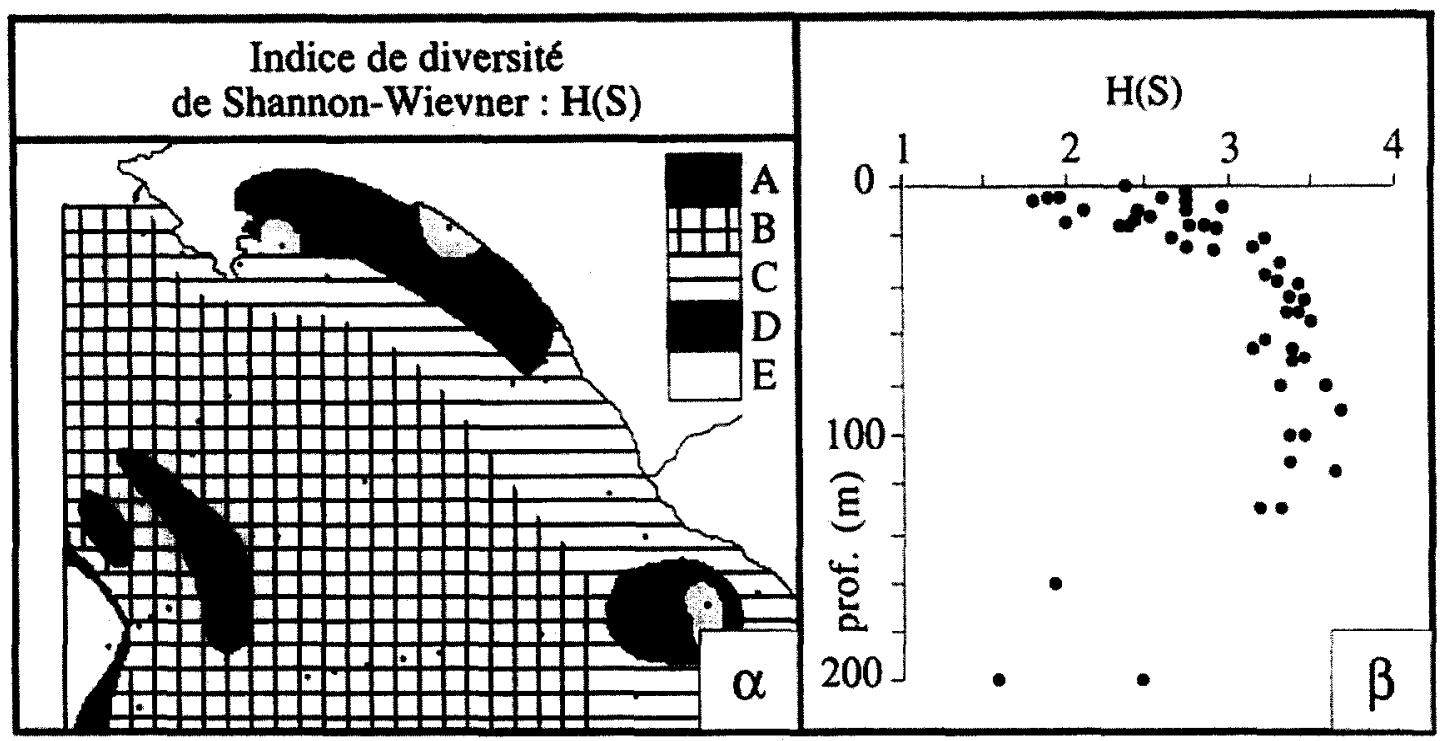

Figure 5. Représentations cartographique $(\alpha)$ et bathymétrique $(\beta)$ de l'indice de diversité de Shannon-Wiener : H(S):(A) $\geq 3,5 ;(B)$ de 3 à 3,$5 ;$ (C) de 2,5 à $3 ;$ (D) de 2 à 2,$5 ;(E)<2$.

Figure 5. Geographic $(\alpha)$ and depth distributions $(\beta)$ of the Shannon-Wiener diversity index: H(S): (A) $\geq 3.5 ;$ (B) 3 to $3.5 ;$ (C) 2.5 to 3 ; (D) 2 to $2.5 ;(\mathrm{E})<2$.

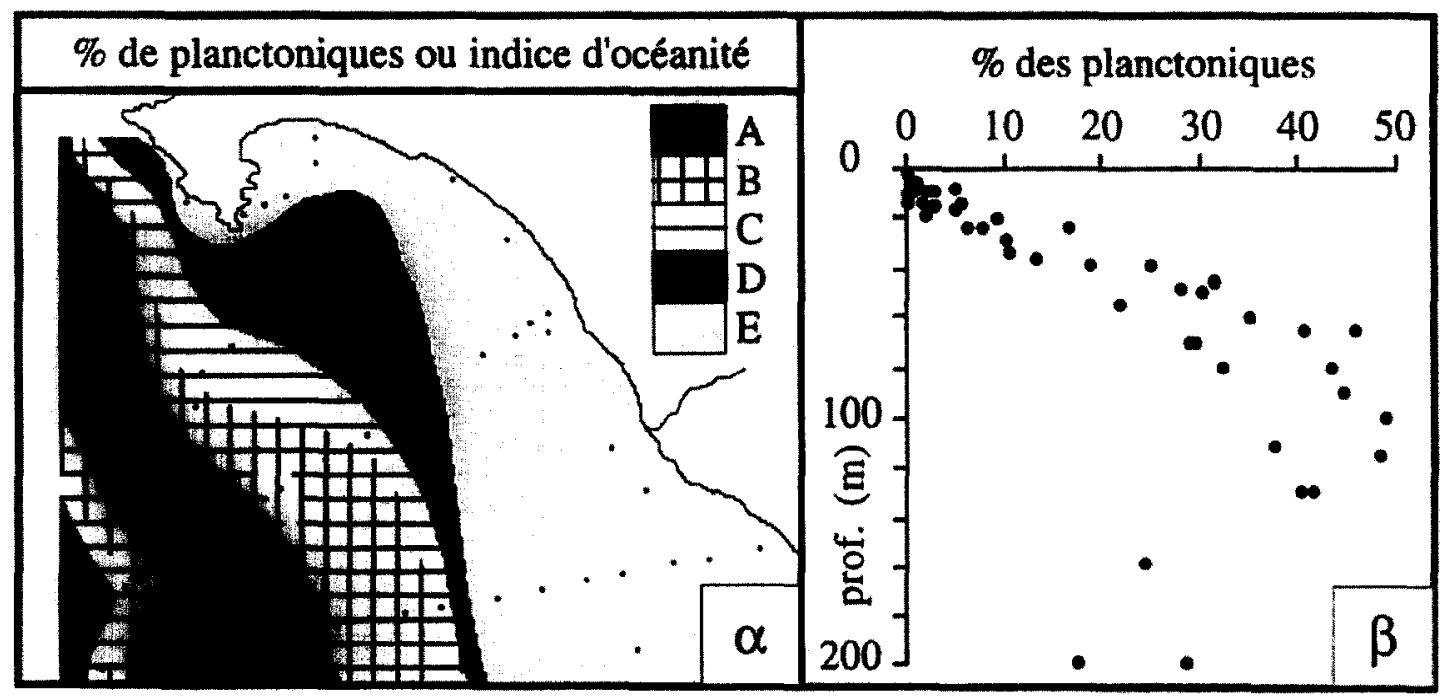

Figure 6. Représentations cartographique $(\alpha)$ et bathymétrique $(\beta)$ du pourcentage de planctoniques : $(A)$ de 40 à $50 \% ;(B)$ de 30 à $40 \%$; (C) de 20 à $30 \%$; (D) de 10 à $20 \%$; (E) $<10 \%$.

Figure 6. Geographic $(\alpha)$ and depth distributions $(\beta)$ of the planktonic percentages: (A) 40 to $50 \%$; (B) 30 to $40 \%$; (C) 20 to $30 \%$; (D) 10 to $20 \% ;(\mathrm{E})<10 \%$. 


\section{DISCUSSION}

\subsection{Taxonomie}

Les problèmes taxonomiques portant sur les 19 espèces dominantes ont déjà été abordés par différents auteurs [5, $13,16]$.

\subsection{Indice de diversité [H (S)]}

L'indice de diversité de Shannon-Wiener est généralement supérieur à 2,1 (figure 5), comme dans les milieux marins « normaux » $[18]$.
Ces valeurs sont les plus faibles lorsque les assemblages sont largement dominés par une ou deux espèces: 1) C. gerthi et $E$. fichtellianum en zone côtière $(0-25 \mathrm{~m})$ où l'énergie des houles est la plus forte ; 2 ) C. laevigata au-delà de $140 \mathrm{~m}$ où les températures sont les plus basses. Les fréquences élevées de cette dernière espèce (jusqu'à $65 \%$ de l'assemblage total) ont également été signalées au Maroc (jusqu'à $45 \%$ ) ainsi qu'au Portugal (jusqu’à $75 \%)[13,16]$.

Les valeurs maximales de l'indice de diversité $(>3,5)$ entre 80 et $115 \mathrm{~m}$, peuvent s'expliquer par la coexistence d'espèces du plateau interne et de celles du plateau externe (tableau II).

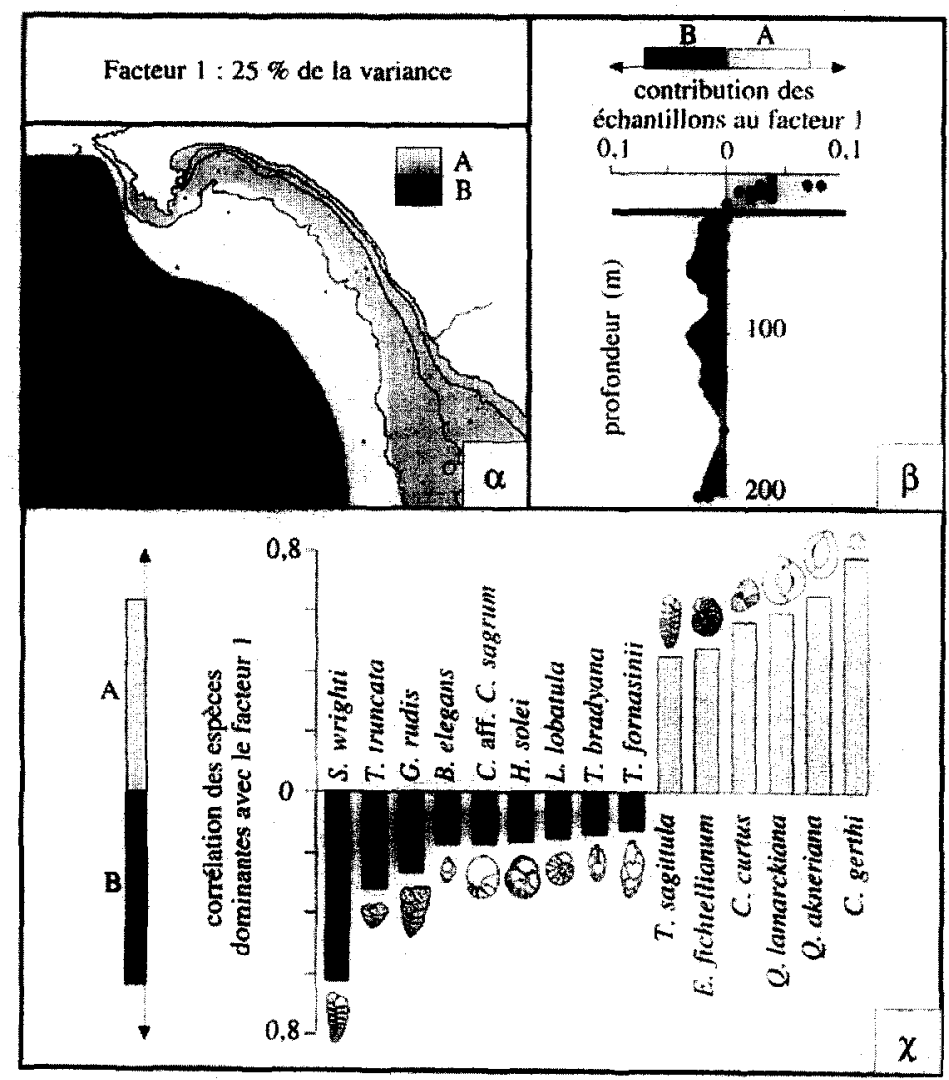

Figure 7. Premier facteur déterminé par l'analyse factorielle. Répartitions cartographique $(\alpha)$ et bathymétrique $(\beta)$ des échantillons contribuant à la détermination du facteur; espèces dominantes corrélées au facteur $(\chi):(A)$ association de zone côtière (coordonnées $>0$ sur axe factoriel) ; (B) association du plateau (coordonnées $<0$ sur axe factoriel).

Figure 7. First factor of the factor analysis; geographic $(\alpha)$ and depth distributions $(\beta)$ of the samples contributing to the factor determination; dominant species correlated to the factor $(\chi)$. (A) coastal zone association (coordinates $>0$ on the factorial axis); (B) shelf association (coordinates $<0$ on the factorial axis). 


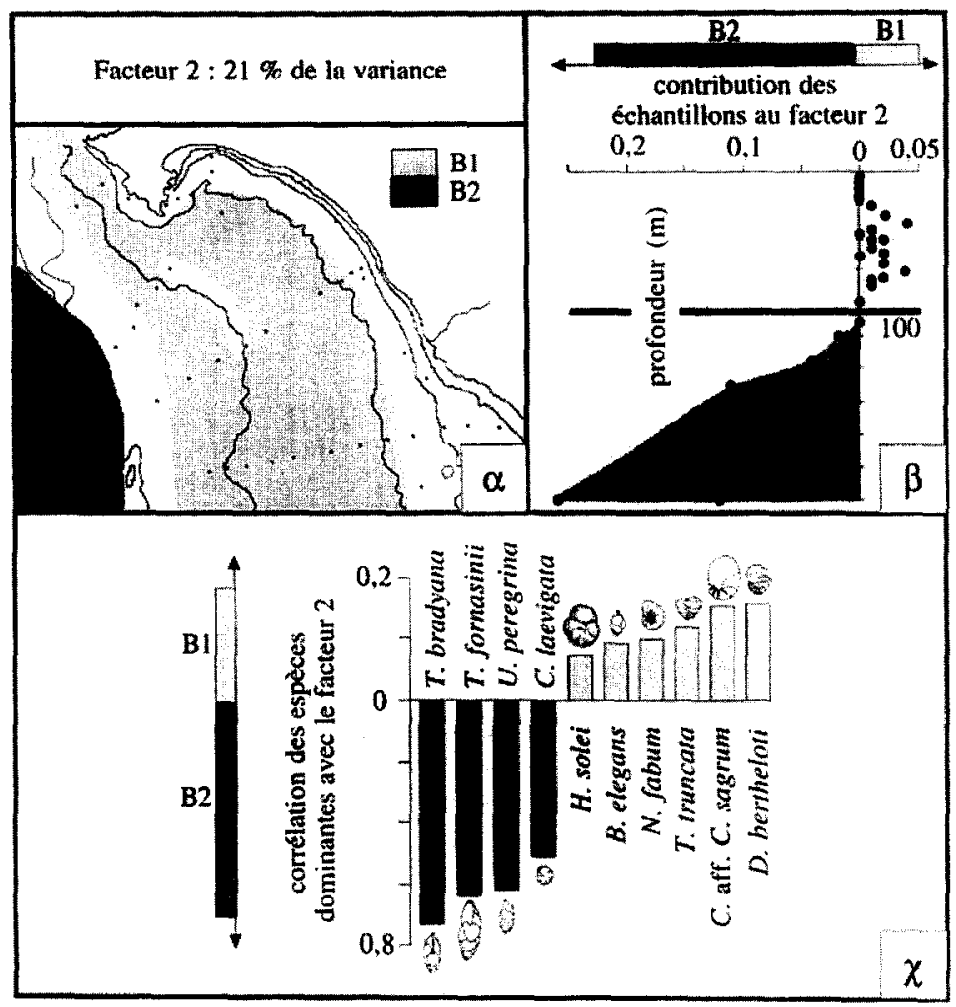

Figure 8. Deuxième facteur déterminé par l'analyse factorielle. Répartitions cartographique $(\alpha)$ et bathymétrique $(\beta)$ des échantillons contribuant à la détermination du facteur ; espèces dominantes corrélées au facteur $(\chi):(B)$ ) association du plateau interne (coordonnées $>0$ sur axe factoriel) ; (B2) association du plateau externe (coordonnées $<0$ sur axe factoriel).

Figure 8. Second factor of the factor analysis; geographic $(\alpha)$ and depth distributions $(\beta)$ of the samples contributing to the factor determination; dominant species correlated to the factor $(\chi)$ : (B1) inner shelf association (coordinates $>0$ on the factorial axis); (B2) outer shelf association (coordinates $<0$ on the factorial axis).

\subsection{Pourcentages de planctoniques}

Sur la zone côtière du Sénégal, les faibles valeurs de ce pourcentage peuvent être liées : 1) à l'énergie des houles et des courants qui réduisent le dépôt des tests planctoniques ; 2) à l'importance des écarts thermiques saisonniers qui limitent le développement de ces formes généralement sténothermes. L'augmentation régulière du pourcentage de planctoniques depuis les faibles profondeurs jusqu'à $100 \mathrm{~m}$ peut donc s'expliquer par la diminution de l'énergie ainsi que par celle des écarts thermiques saisonniers [13]. Cette augmentation peut également résulter du dépôt des tests d'espèces qui vivent à des profondeurs plus grandes [19]. La diminution du pourcentage de planctoniques sur le plateau externe (figure 6) a également été signalé en Algarve ou au Maroc [12, 13, 16]. Cette diminution résulterait probablement d'une augmentation de la productivité benthique induite par les upwellings.

\subsection{Influence de la profondeur et de la température}

\subsubsection{Sur les coupures fauniques}

La coupure faunique la plus nette (vers $25 \mathrm{~m}$; figure 7 ) correspond à la limite supérieure de la thermocline (isotherme $25^{\circ} \mathrm{C}$ ) au-dessus de laquelle les écarts thermiques saisonniers sont maximaux $\left(>10^{\circ} \mathrm{C}\right)$. L'influence de la température sur les coupures fauniques a également été signalée dans d'autres régions mais à des profon- 


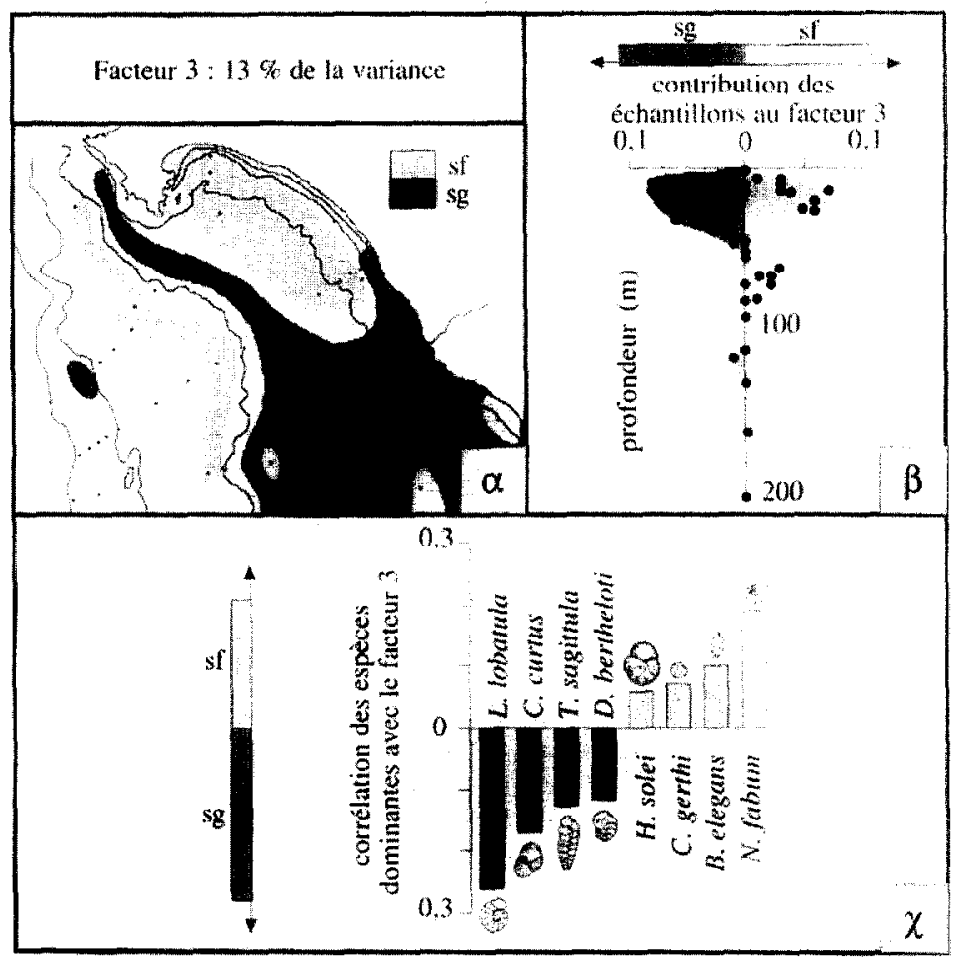

Figure 9. Troisième facteur déterminé par l'analyse factorielle. Répartitions cartographique $(\alpha)$ et bathymétrique $(\beta)$ des échantillons contribuant à la détermination du facteur; espèces dominantes corrélées au facteur $(\chi):($ Sf) association des sables fins (coordonnées $>0$ sur axe factoriel) ; (sg) association des sables grossiers (coordonnées $<0$ sur axe factoriel).

Figure 9. Third factor of the factor analysis; geographical $(\alpha)$ and depth distributions $(\beta)$ of the samples contributing to the factor determination; dominant species correlated to the factor $(\chi)$ : (sf) fine-grained association (coordinates $>0$ on the factorial axis); (sg) coarse-grained association (coordinates $<0$ on the factorial axis).

deurs plus importantes $(50-70 \mathrm{~m})$ [3] ; d'autres facteurs tels que la salinité ou la présence de végétaux, pourraient également être responsables de coupures fauniques peu profondes (entre 0 et $40 \mathrm{~m}$ de profondeur).

La deuxième coupure faunique (vers $85 \mathrm{~m}$; figure 8 ) a été signalée sur d'autres plateaux continentaux de l'Atlantique sud $[3,17]$. Au sud de Dakar, sa position correspond à celle de l'isotherme $15^{\circ} \mathrm{C}$, au-dessous de laquelle les écarts thermiques saisonniers sont minimaux (figure 2 et 8). Selon Murray [17] et Blanc-Vernet [3], cette coupure peut être expliquée par le remplacement d'espèces eurythermes par les espèces plus sténothermes du plateau externe. La température, elle-même liée à la profondeur, semble donc jouer un rôle déterminant sur la localisation de ces deux coupures fauniques.

\subsubsection{Sur les espèces}

Au Sénégal comme dans d'autres secteurs des côtes atlantiques $[16,18]$, certaines espèces dominantes du plateau externe ( $C$. laevigata, $T$. hradyana et $U$. peregrina) ont une répartition qui est surtout liée aux temperatures relativement basses de ce secteur $\left(<15-16^{\circ} \mathrm{C}\right)$.

En revanche, d'autres espèces dominantes de la zone côtière (C. gerthi; E. fichtellianum) semblent avoir un comportement qui differe selon les sites étudiés : elles seraient eurythermes au sud de Dakar (température de 16 à $27^{\circ} \mathrm{C}$ ) et plutôt sténothermes au Maroc où les variations thermiques saisonnières sont relativement faibles (température de 13 à $17^{\circ} \mathrm{C}$; [16]). En fait, le comportement écologique de ces espèces ne pourra être clairement 
établi qu'à partir d'études basées sur le suivi des biocénoses pendant au moins un cycle annuel.

\subsubsection{Influence du substrat sur la répartition des espèces}

Certaines relations observées dans cette étude entre les espèces et la nature du sédiment ont déjà été signalées dans la littérature ; c'est le cas pour l'affinité de $N$. fabum avec les sables fins ou encore pour celle de L. lobatula avec les sables grossiers et les végétaux sur lesquels elle se fixe $[10,16,18]$. Cette dernière espèce est associée dans le sédiment avec des débris d'autres organismes qui peuvent se fixer sur des rochers, des graviers ou encore des végétaux (balanes; bryozoaires). En ce qui concerne les végétaux, les données disponibles sur le plateau continental sénégalais sont insuffisantes pour permettre d'établir leur influence directe sur la répartition de L. Lobatula.

L'affinité de $G$. rudis avec les sables grossiers a été signalée sur certains sites des côtes atlantiques $[16,18]$ mais également à partir de l'étude d'une radiale au sud de Dakar [25]. Cette affinité ne semble cependant plus aussi évidente après l'examen de la totalité des échantillons prélevés sur le plateau continental sénégalais.

\subsubsection{Influence du transport}

Seuls les tests frais sans minéralisation et sans marque d'usure ont été retenus pour les comptages. D'après Pujos [23], ces caractéristiques correspondent aux tests issus des biocénoses ou des thanatocénoses non transportées («niveau d'usure $1 »)$. Notre choix de ne compter que les tests frais permet donc d'éliminer en grande partie les effets du transport.

Malgré ces précautions, de rares individus transportés ou reliques ont pu être pris en compte dans les comptages ; c'est notamment le cas pour Ammonia tepida ou pour Cribroelphidium gunteri (planche I, 11). La présence de ces deux espèces à proximité des côtes pourrait en fait indiquer : 1) un transport depuis les lagunes côtières où ces espèces sont bien représentées ; 2 ) ou bien l'existence d'une faune relique issue de lagunes holocènes formées au cours de la dernière transgression [26].

\section{CONCLUSION}

Ce travail complète les connaissances sur les foraminiferres benthiques du plateau continental sénégalais où très peu d'études ont été menées jusqu'à maintenant. L'étude de la répartition des 19 espèces dominantes (cartes, analyse factorielle), permet de déterminer deux principales coupures fauniques $(25$ et $85 \mathrm{~m}$ ) et trois associations liées à la bathymétrie : association de zone côtière $(0-25 \mathrm{~m})$, de plateau interne $(25-85 \mathrm{~m})$ et de plateau externe $(85-200 \mathrm{~m})$. Ce découpage faunique semble être lié à la température ainsi qu'aux écarts thermiques saisonniers des eaux de fond. L'influence du substrat sur la répartition de ces organismes est surtout nette en zone côtière et sur le plateau interne où les ensembles sédimentaires sont hétérogènes ; ils permettent de distinguer les espèces de sables fins et celles qui sont plus fréquentes dans les sables grossiers. L'influence des upwellings sur le plateau externe (remontées d'eaux profondes riches en éléments nutritifs) est surtout marquée par la diminution du pourcentage des planctoniques et celle de l'indice de diversité ; elle correspond à l'augmentation de la productivité benthique d'une population peu diversifiée.

\section{Remerciements}

Les auteurs remercient $\mathrm{M}$. Lesourd du service commun de microscopie électronique (SCME) de l'université d'Angers, pour la réalisation des clichés au microscope électronique à balayage. Ils remercient également J.-P. Masse de l'université de Marseille qui a mis les échantillons à leur disposition.

\section{RÉFÉRENCES}

[1] Barusseau J.P., Analyse sédimentologique des fonds marins de la « Petite Côte " (Sénégal), Doc. Scientifique ISRA/CRODT 94 (1984) 1-23.
[2] Basov I.A., Quantitative distribution of benthonic foraminifers on the north-west African shelf, Oceanology 15 (2) (1975) 325-329. 
[3] Blanc-Vernet L., Répartition bathymétrique des foraminifères benthiques sur la plate-forme continentale, Géol. Méditerranéenne 15 (1) (1988) 5-12.

[4] Culver S.J., Buzas M.A., Recent benthic foraminiferal provinces on the Atlantic continental margin of North America, J. Foram. Res. 11 (1981) 217-240.

[5] Debenay J.P., Basov I.A., Distribution of recent benthic Foraminifera on the West African shelf and slope ; A synthesis, Rev. de Paléobiologie 12 (2) (1993) 265-300.

[6] Debenay J.P., Redois F., Distribution of the 27 dominant species of shelf benthic foraminifers on the continental shelf, north of Dakar (Senegal), Marine Micropaleontlogy 29 (1997a) 237-255.

[7] Debenay J.P., Redois F., Recent Foraminifera of the northern continental shelf of Senegal, Revue de Micropaléontologie 40 (1) (1997b) 15-38.

[8] Domain F., Description de la sédimentation fine et des formations rocheuses du plateau continental ouest-africain de $17^{\circ} \mathrm{N}$ à $12^{\circ} \mathrm{N}$, Bull. Liaison ASEQUA 50 (1977) 11-22.

[9] Haake F.W., Bentische Foraminiferen in OberflächenSedimenten und Kernen des Ostatlantiks vor Senegal/Gambia (Westafrika), « Meteor » Forsch.-Ergebnisse C, 32 (1980) 129.

[10] Kouyoumontzakis G., Les associations de foraminiferes benthiques du plateau continental congolais ; une radiale au large de la lagune Conkouati, Tethys 10 (2) (1981) 121-128.

[11] Kouyoumontzakis G., Les Amphisteginidae (Foraminifera) du plateau continental congolais dans le cadre de la marge ouest africaine, Rev. Micropal. 27 (3) (1984) 196-208.

[12] Lévy A., Mathieu R., Poignant A., Rosset-Moulinier M., Ubaldo M.L., Ambroise D., Recent Foraminifera from the continental margin of Portugal, Micropaleontology 39 (1) (1993) 75-87.

[13] Lévy A., Mathieu R., Poignant A., Rosset-Moulinier M., Ubaldo M.L., Lebreiro S., Foraminifères actuels de la marge continentale portugaise, inventaire et distribution, mem. do Instituto Geol. e Mineiro 32 (1995) 116 p.

[14] Loeblich A.R., Tappan H., Foraminiferal Genera and their classification, Van Nostrand Reinhold, vol. 2, 1988, 970p.

[15] Masse J.P., Contribution à l'étude des sédiments actuels du plateau continental de la région de Dakar-Sénégal (essai d'analyse de la sédimentation biogène), rapp. lab. géol. Dakar $23(1968) 1-84$.

[16] Mathieu R., Sédiments et foraminifères actuels de la marge continentale atlantique du Maroc, thèse sci. univ. Paris-VI, 8614 (1986) $420 \mathrm{p}$.

[17] Murray J.W., Distribution and ecology of living benthic Foraminiferids, Heinemann Educational Books, (1973) 274 p.

[18] Murray J.W., Ecology and paleoecology of benthic Foraminifera, Longman Scientific and Technical, (1991a) 397 p.

[19] Murray J.W., Ecology and Distribution of Benthic Foraminifera, in : Biology of the Foraminifera, Lee J.J. and Anderson O.R., (éd.) Academic Press, (1991b) pp. 221-253.

[20] Patterson T., Fishbein E., Re-examination of the statistical methods used to determine the number of point counts needed for micropaleontological quantitative research, J. Paleont. 63. 2 (1989) 245-248.

[21] Phleger F.B., Ecology and distribution of recent Foraminifera, The Johns Hopkins Press, (1960) 297 p.

[22] Pinson-Mouillot J., Les environnements sédimentaires actuels et Quaternaires du plateau continental sénégalais (Nord de la presqu'île du Cap Vert), thèse sci. univ. Bordeaux-I, (1554) (1980) $142 \mathrm{p}$.

[23] Pujos M., Mise en évidence des biocénoses, faunes déplacées, paléothanatocoenoses de foraminifères benthiques sur un plateau continental : applications à la zone ouest-Gironde (golfe de Gascogne), Bull. Soc. géol. de France (7) XIII 3-4 (1971) 251-256.

[24] Rebert J.P., Hydrologie et dynamique des eaux du plateau continental sénégalais, Doc. Scientifique CRODT. 89 (1983) 97.

[25] Redois F., Foraminifères benthiques d'une radiale au large de Dakar, influence de la profondeur, du substrat et des upwellings, in : géologie de l'Afrique et de l'Atlantique sud, actes colloques Angers 1994, Jardiné S., Klasz I. de, Debenay J.P.; (éd.) Bull. Centres Rech. Explor.-Prod. Elf Aquitaine, Mem. 16 (1996) 343-349.

[26] Riffault A., Les environnements sédimentaires actuels et Quaternaires du plateau continental sénégalais (sud de la presqu'île du Cap Vert), Sénégal, thèse sci. univ. Bordeaux-I, 1561 (1980) $143 \mathrm{p}$. 